\title{
VALUE OF FECAL CALPROTECTIN AS A NON-INVASIVE MARKER DIFFERENTIATING ORGANIC FROM FUNCTIONAL BOWEL DISEASES Clinical and histopathological study
}

\author{
Yasser S. Sheta*, Ihab M. Salem*, Waleed A. Ismail* Abeer M. Hafez** \\ From the departments of Internal medicine*, Pathology** Faculty of Medicine, Zagazig University
}

\begin{abstract}
Background: Bowel diseases are very common, most of them are functional not organic in nature but clinicians suffer a lot in differentiating between the two categories especially the management is completely different. The symptoms of functional bowel diseases can be very similar to organic inflammatory bowel diseases like ulcerative colitis. In many cases the definitive diagnosis needs invasive procedures including lower colonoscopy and biopsy for histopathological documentation. Aim of the work: To evaluate the clinical value of fecal calprotectin as a non invasive marker differentiating organic from functional bowel diseases in correlation with endoscopic and histopathological examinations. Subjects and methods: The study included 40 patients, selected to represent 2 groups: group (I) included 20 patients with ulcerative colitis (UC) proved by colonoscopy and histopathology who further subdivided into sub-groups according to activity and site of involvement; and group (II) included 20 patients who had symptoms suggestive of irritable bowel syndrome (IBS) and in whom colonoscopy was found to be normal served as control. All participants were subjected to thorough history taking, physical examination and routine laboratory investigations. Calprotectin in feces was measured using the enzyme-linked immunosorbent assay (ELISA).Results: Fecal calprotectin concentration in the patients with UC was significantly higher than in patients with IBS (Mean values \pm SD; $201.7 \pm$ $46.7 \mu \mathrm{g} / \mathrm{g}$ vs, $22.3 \pm 10.1 \mu \mathrm{g} / \mathrm{g}, \mathrm{P}<0.01)$. A significant difference was also found in the patients with active UC in comparison to UC patients without sign of activity (Mean values $\pm \mathrm{SD} ; 220.2 \pm 31.1 \mu \mathrm{g} / \mathrm{g} \mathrm{vs}, 167.3 \pm 23.9 \mu \mathrm{g} / \mathrm{g}$, $\mathrm{P}<0.05)$. The sensitivity of fecal calprotectin at cut off value $195.5 \mu \mathrm{g} / \mathrm{g}$; as activity marker of UC was $82.2 \%$ while its specificity was $85.7 \%$., Positive predictive value was $90.1 \%$ and negative predictive was $66.6 \%$. Conclusion: Fecal calprotectin can differentiate with great acceptance between organic and functional bowel diseases and can be used as a rational fecal marker for intestinal inflammation in clinical practice. This kind of marker is relatively precise, simple and noninvasive and could give a crude idea about the activity of the lesions.

Key words: Fecal calprotectin; Ulcerative colitis; Enzyme-linked immunosorbent assay.
\end{abstract}

\section{INTRODUCTION}

I rritable bowel syndrome (IBS), one of the most common gastrointestinal disorders seen in primary health care and the commonest among functional bowel disorders (1).It is characterized as most of functional bowel disorders with chronic lower abdominal pain/discomfort, distention and changes in bowel habits (2). Several studies specified that there is a female predominance and the prevalence is $10-20 \%$ in the Western world (3). Despite the benign nature of IBS; the symptoms can be very similar to organic inflammatory bowel disease which makes a lot of troubles to clinicians especially in primary care clinics. In many cases the definitive diagnosis needs invasive procedures including lower endoscopies and biopsy for histopathological documentation (4). Inflammatory bowel disease (IBD) is a chronic condition characterized by recurrent episodes of inflammation in the gastrointestinal tract and includes crohn's disease (CD) and ulcerative colitis (UC). Patients with IBD experience diarrhea, abdominal pain and cramps, disrupted digestion, rectal bleeding, weight loss and a substantial personal burden (56 ). The precise etiology of IBD is remained mainly unknown but is thought to be a complex interaction of immunological, genetic and environmental (such as enteric microflora) factors (7-8). Calprotectin is a member of the Ca-binding S100 family of proteins, a heterogeneous complex of $\mathrm{S} 100 \mathrm{~A} 8 / \mathrm{S} 100 \mathrm{~A} 9$ proteins. It is present in neutrophils and monocytes and is released by activation of these cells in plasma, urine, stools and other media as a consequence of disease activity (9). Several studies reported that fecal calprotectin (FC) level is associated with invasive markers of gut inflammation such as 99Tc-labeled white cell scans and endoscopic and histologic inflammation scores (10).Recent studies showed FC is a specific, sensitive, non-invasive, cheap and accessible marker for gut inflammation (1112). The aims of the present study were to evaluate the clinical value of fecal calprotectin as a non invasive marker in differentiating organic from functional bowel diseases in correlation with endoscopic and histopathological examinations in Egyptian patients.

\section{SUBJECTS AND METHODS}

This study was carried out in the gastroenterology unit of Internal Medicine and Histopathology departments, Faculty of Medicine, Zagazig University in Egypt. All the preliminary participants in the study referred to diagnostic colonoscopy unit for evaluation of chronic lower abdominal pain plus mixture of vague symptoms 
of abdominal distention, diarrhea, sense of incomplete defecation and change of bowel habits unresponsive to routine dietary and symptomatic treatment for at least six months from June 2009 till May 2010. Colonoscopic examination was done for all studied patients after standard bowel preparation using a Pentax videoscope EC-3440F. According to colonoscopic picture, they divided into two groups: Group I: included 29 patients with colonoscopic evidence of intestinal inflammation. Twenty three fulfill the endoscopic criteria of UC (Loss of the vascular appearance of the colon, erythema and friability of the mucosa, superficial ulceration and pseudopolyps) (13). Three fulfill the criteria of Crohn's disease (a patchy distribution of disease, with involvement of the colon or ileum, but not the rectum and cobblestone-like appearance representing areas of ulceration separated by narrow areas of healthy tissue) (14).The remaining three cases showed Table 1 evidence of inflammation in non specific manner (non-specific colitis).As the number of patients of Crohn's disease and those of non specific colitis were very small and cannot be relied upon statistically, they excluded from the study. Also three patients from UC group were excluded because they had concomitant chronic illness: bronchial asthma requiring corticosteroid (two patients) and moderate chronic kidney disease (one patient).

The remaining twenty patients with UC, the group of organic inflammatory bowel disease; their age ranged from 17 to 52 years (mean $\pm \mathrm{SD}=34.5 \pm$ 11.6 years). Those patients subdivided into two subgroups according to site of involvement either rectum alone (12 patients) or rectum and ascending colon (8 patients). Biopsy is taken from all patients in this group for further histopathological documentation. Criteria of diagnosis of UC are shown in table 1(15).

\begin{tabular}{lll}
\hline Architecture & Inflammatory & Miscellaneous \\
\hline$\bullet \quad$ Severe crypt architectural & $\bullet \quad$ Heavy diffuse & $\bullet \quad$ Increased intensity of the \\
distortion & transmucosal lamina & alterations towards the distal \\
$\bullet \quad$ Severe widespread & propria cell increase & colon \\
decreased crypt density & $\bullet \quad$ Diffuse basal & $\bullet \quad$ Severe mucin depletion \\
$\bullet \quad$ Frankly villous surface & plasmacytosis & $\bullet \quad$ Paneth-cell metaplasia distal \\
& & to the hepatic flexure
\end{tabular}

The patients' disease activities were assessed according to the Sutherland criteria (16) in which a score of more than two was considered to indicate the active stage of the disease dividing the patients into 2sub groups: either with activity (13 patients) or without activity (7 patients).

Group II: included 58 patients without endoscopic evidence of intestinal inflammation and considered as functional bowel disease (IBS).After application of the exclusion criteria which were presence of malignancy, immunosuppressive therapy; active gastrointestinal bleeding, pregnancy and any other chronic illness necessitate long term drug therapy or cause immune modulation. Many of them were excluded from the study and we choosed the best twenty ages and sex matched patients to the members of group I. Patients' age ranged 19-49 years (mean $34.1 \pm 9.8$ years).

A sample of stool was collected from each patient before colonoscopy and kept in $-20^{\circ} \mathrm{C}$ until the time of analysis. Mean time difference between the time of specimen collection and analysis was 4 months. Analysis was performed using an ELISA kit for calprotectin (Buhlmann Co., Switzerland).
Briefly, the samples were thawed, and then $50 \mathrm{mg}$ of each sample was added to $2.5 \mathrm{ml}$ extraction buffer, provided in the kit, mixed well and centrifuged at $3500 \mathrm{rpm}$ for 15 minutes. The supernatant was used for ELISA sandwich test. Approval was granted by the local and regional ethics committees, and participants gave informed written consent.

\section{Statistical Analysis}

The results were presented as mean \pm standard deviation (S.D.). Statistical comparisons of individual groups were based on unpaired Student's t-test. The gender ratio was compared with $\chi 2$ test. $\mathrm{P}$ value is considered significant at $\leq$ 0.05 level, highly significant at $\leq 0.01$ and non significant at $>0.05$.

\section{RESULTS}

The study included 40 subjects, selected to represent 2 groups: group (I) included 20 patients with UC proved by colonoscopy and histopathology represent patients with organic inflammatory bowel diseases and further subdivided into sub-groups according to activity and site of involvement, figure 1-2 show active $\mathrm{UC}$ and figure 3 shows UC without acute activity, 
group (II) included 20 patients who had symptoms found to be normal. suggestive of IBS and in whom colonoscopy was

Figure 1:

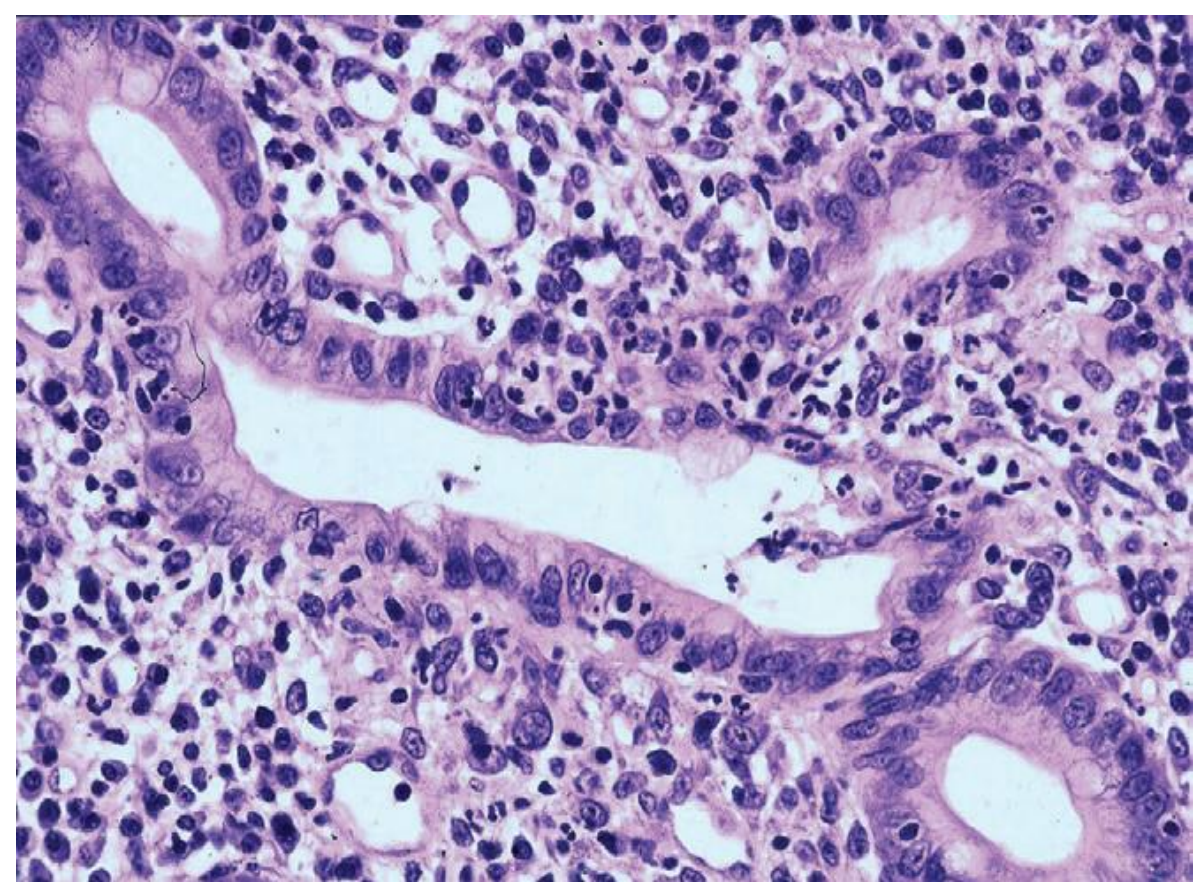

The presence of neutrophilic polymorphs in the lamina propria, surface epithelium, crypt epithelium (cryptitis) defines disease activity(H\&E .40).

Figure 2:

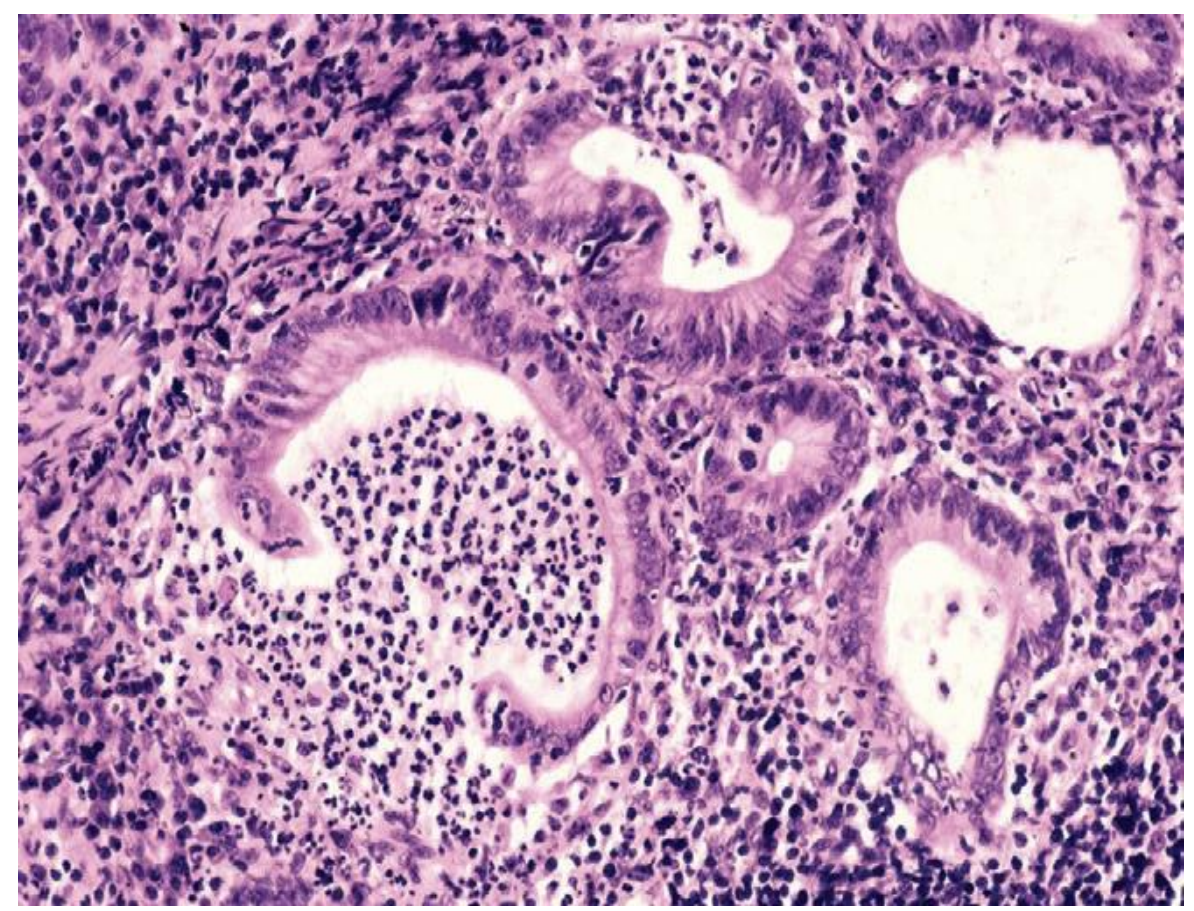

Inflammatory infiltration: cryptic abscess (H\&E .40). 
Figure 3:

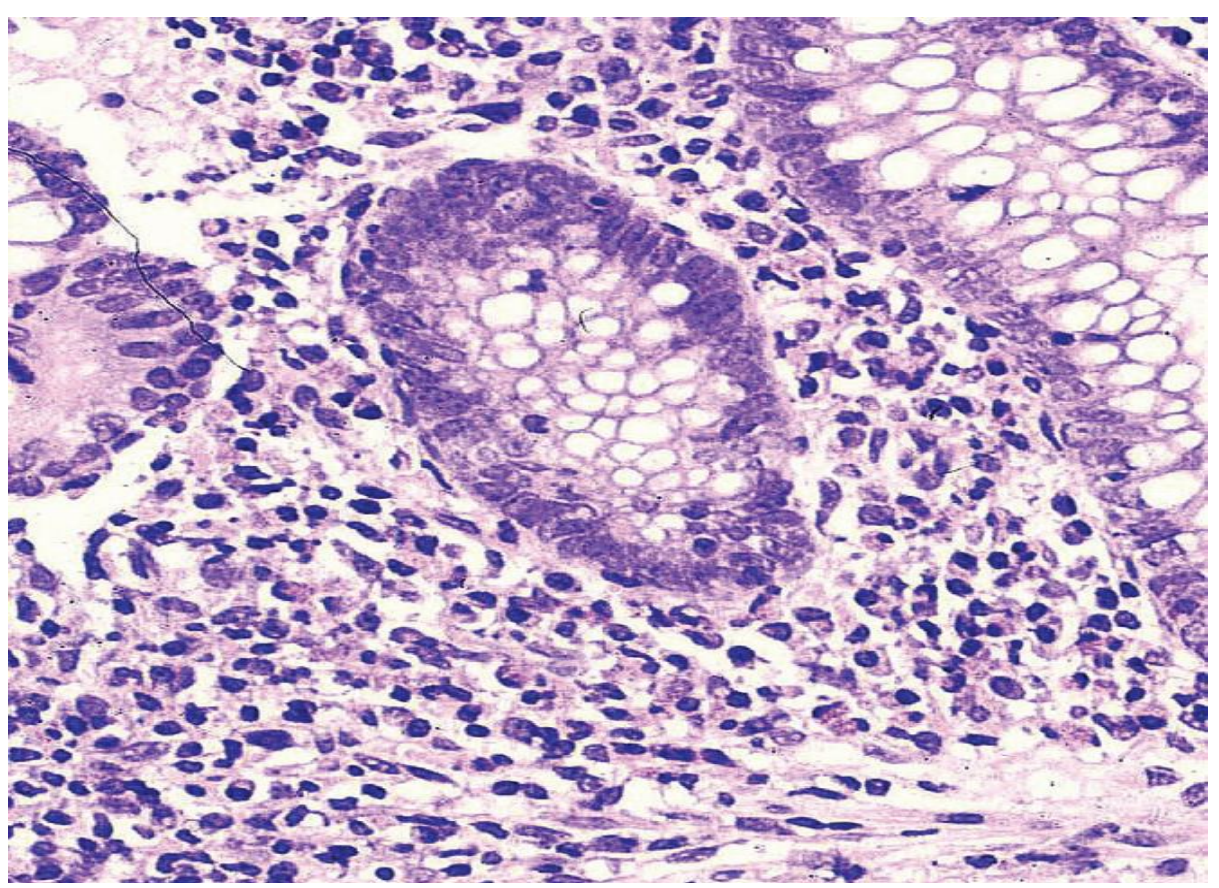

Presence of a large number of chronic inflammatory cells \& plasma cells between the bottom of the crypts and the muscularis mucosae without evidence of neutrophils indicates chronic inflammatory process without acute activity (H\&E. 40).

Both groups were age and sex matched and no significant difference $\mathrm{p}>0.05$ table 2 .

(Table 2) Clinical characteristics of the patients

\begin{tabular}{llll}
\hline & Group I $(\boldsymbol{n}=\mathbf{2 0})$ & Group II $(\boldsymbol{n}=\mathbf{2 0})$ & $\boldsymbol{p}$ \\
\hline Age(years) & $34.5 \pm 11.6$ & $34.1 \pm 9.8$ & NS \\
\hline Males/females & $\mathbf{1 1 / 9}$ & $\mathbf{1 0 / 1 0}$ & NS \\
\hline
\end{tabular}

Very highly significant difference; as regard the mean value of fecal calprotectin level in patients with UC in comparison to the mean value of patients with functional IBS (Mean values $\pm \mathrm{SD} ; 201.7 \pm 46.7 \mu \mathrm{g} / \mathrm{g}$ vs, 22.3 $\pm 10.1 \mu \mathrm{g} / \mathrm{g}, \mathrm{P}<0.01)$ figure 4 .

No significant difference of fecal calprotectin level between patients with ulcerative proctitis and patients with ulcerative proctatitis and ascending colitis (Mean values \pm SD; $196.3 \pm 39.9 \mu \mathrm{g} / \mathrm{g}$ vs, $209.9 \pm 42.6 \mu \mathrm{g} / \mathrm{g}$, $\mathrm{P}>0.05$ ) figure 4 .

While there is significant difference as regard fecal calprotectin level in UC patients showed signs of activity when compared to UC patients without activity (Mean values $\pm \mathrm{SD} ; 220.2 \pm 31.1 \mu \mathrm{g} / \mathrm{g} \mathrm{vs}, 167.3 \pm 23.9$ $\mu \mathrm{g} / \mathrm{g}, \mathrm{P}<0.05)$ figure 4 .

Figure (4) Comparison of mean values of fecal calprotectin level in different groups and subgroups of the study

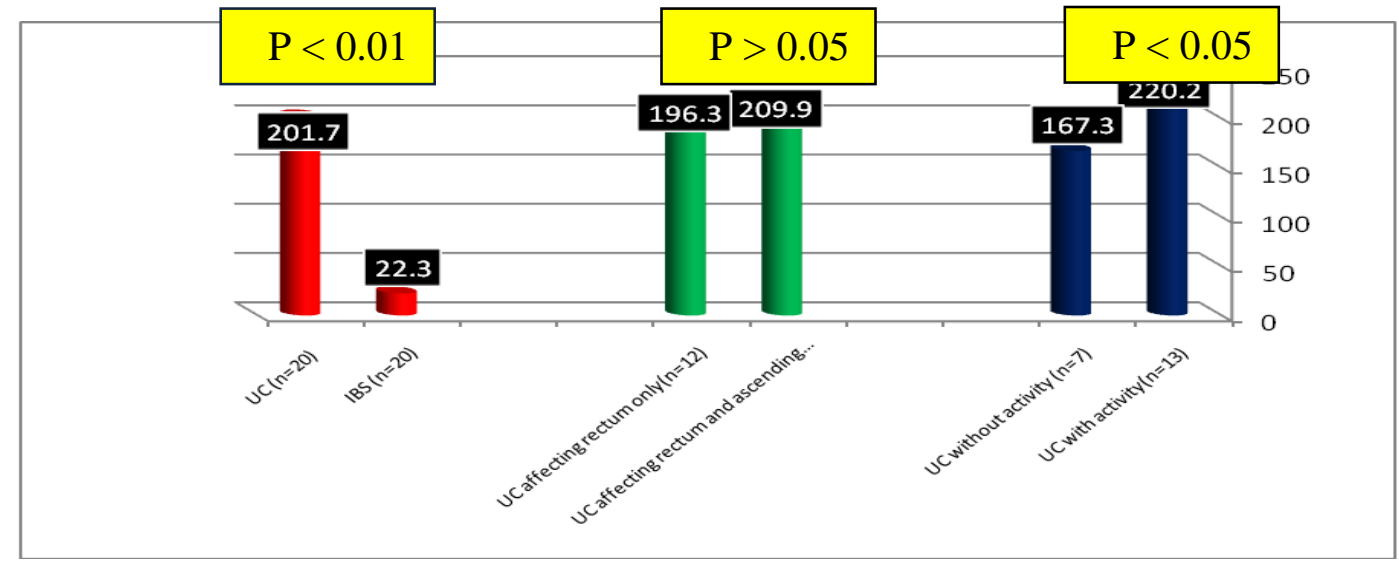


(Table 3) Sensitivity, Specificity, Positive predictive value and Negative predictive value of fecal calprotectin as a marker of UC activity at cut off value $195.5 \mathrm{ug} / \mathrm{g}$

\begin{tabular}{lll}
\hline Sensitivity & Specificity & Positive pred \\
\hline $82.2 \%$ & $85.7 \%$ & $90.1 \%$ \\
\hline \multicolumn{3}{c}{ DISCUSSION }
\end{tabular}

Bowel diseases are very common, most of them are functional not organic in nature but clinicians suffer a lot in differentiating between the two categories especially the management is completely different (1). The symptoms of functional bowel diseases including chronic lower abdominal pain/discomfort, distention and changes in bowel habits can be very similar to organic inflammatory bowel diseases like ulcerative colitis (17). The course of IBD is characterized by periods of relapse and remission because of increases and decreases in mucosal inflammatory activity, respectively (5). An important step in the primary assessment of patients with IBD is measurement of bowel inflammation as it defines the extent and severity of involvement at the beginning of treatment and during monitoring in order to target medical therapies and manage IBD-related complications (18). Many methods of assessing bowel inflammation have been proposed, including laboratory indices and clinical scores that generally are considered nonspecific (10). In many cases the definitive diagnosis needs invasive procedures including lower colonoscopy and biopsy for histopathological documentation (18). It has been suggested that an inflammatory mediator directly released into the gut lumen from the inflammatory process might be an ideal test to detect bowel inflammation in IBD (19). In many studies, fecal calprotectin has been claimed to be a specific, sensitive, non-invasive, cheap and accessible marker for gut inflammation $(11,12$, 17). The fact that calprotectin is stable in room temperature for 5 days makes it more suitable for routine clinical studies (20). In our study, fecal calprotectin levels in organic UC patients were obviously higher than those in IBS and the difference between the two groups was statistically significant $(\mathrm{p}<0.01)$. This finding confirms the results of previous studies; e.g., a study by Tibble et al. (21). In our study despite the marvelous success of fecal calprotectin in distinguishing organic from functional bowel disease but it cannot differentiate between patients with UC as regard site and extent of involvement whatever limited to rectum alone or extend to ascending colon. Also should be reiterated that fecal calprotectin cannot distinguish infectious bowel disease from IBD, so appropriate culture studies should always be performed before diagnosing a patient as IBD when the level of fecal calprotectin is high because in both conditions there is heavy invasion by neutrophils ; the main mother cell of calprotectin (9).In the other hand our results showed significant difference between UC patients according to presence or absence of signs of activity with higher values in active disease which simply explained by same concept that during activity there is huge invasion by neutrophils. These results confirm the results of Jun-Ying Xiang et al (22).which concluded that fecal calprotectin concentration in the patients with active UC was significantly higher than that in the inactive UC. They also found a significant difference was also found in the patients with active UC of mild, moderate and severe degrees but they did not determine certain cut off value of fecal calprotectin differentiating active from inactive UC. In our study; sensitivity and specificity of fecal calprotectin as a separate line differentiating active from inactive UC were $82.2 \%, 85.7 \%$ respectively.

\section{CONCLUSION}

Calprotectin in the patient's feces can differentiate with great acceptance between organic and functional bowel diseases and can be used as a rational fecal marker for intestinal inflammation in clinical practice. This kind of marker is relatively precise, simple and noninvasive and could give a crude but valuable idea about the activity of the lesions. Further studies are needed for determining its value in other organic diseases, its guidance for choosing the best modality of treatment and its use as marker of remission and success of management.

\section{REFERENCES}

1. ECRI Institute. Hotline Response. Serologic Markers for Diagnosis of Irritable Bowel Syndrome.November 2010.

2. Barbara G, Wang B, Stanghellini V, et al. Mast cell-dependent excitation of visceral-nociceptive sensory neurons in irritable bowel syndrome.Gastroenterology 2007;132:26-37.

3. Meucci G, D'Incà R, Maieron R, et al. Diagnostic value of faecal calprotectin in unselected outpatients referred for colonoscopy: A multicenter prospective study. Dig Liver Dis. 2010 Mar; 42(3):191-5.

4. Mehrjardi A, SaberAfsharian M, Mirskandari M, et al.Comparison of fecal calprotectin level in 
inflammatory bowel disease and irritable bowel syndrome. Govaresh \Vol. 14, No.4, winter 2010; 275-278.

5. Aomatsu T, Yoden A, Matsumoto K, et al. Fecal calprotectin is a useful marker for disease activity in pediatric patients with inflammatory bowel disease. Dig Dis Sci. 2011 Aug; 56(8):2372-7.

6. Targan SR, Karp LC. Defects in mucosal immunity leading to ulcerative colitis. Immunol Rev 2005; 206:296-305.

7. Bouma G, StroberW. The immunological and genetic basis of inflammatory bowel disease. Nat Rev Immunol 2003; 3:521-33.

8. Ho GT, Lee HM, Brydon G, et al. Fecal calprotectin predicts the clinical course of acute severe ulcerative colitis. Am J Gastroenterol 2009 Mar; 104(3):673-8

9. Roseth AG, Fagerhol MK, Aadland E, et al. Assessment of the neutrophil dominating protein calprotectin in feces: A methodologic study. Scand J Gastroenterol1992; 27:793-8.

10. Canani RB, de Horatio LT, Terrin G,et al . Combined use of noninvasive tests is useful in the initial diagnostic approach to a child with suspected inflammatory bowel disease. J Pediatr Gastroenterol Nutr 2006; 42:9-15.

11. Sidler MA. Leach ST, S.Day AS. Fecal S100A12 and fecal calprotectin as noninvasive Markers for inflammatory bowel disease in children. Inflamm Bowel Dis 2008; 14:359-66.

12. Jellema P, van Tulder MW, van der Horst HE, et al. Inflammatory bowel disease: a systematic review on the value of diagnostic testing in primary care. Colorectal Dis. 2011 Mar; 13(3):239-5416.

13. Kornbluth A, David Bs, Ulcerativ. colitis practice guidelines in adults (update): American College of Gastroenterology, Practice Parameters Committee. Am J Gastroenterol 2004 July;99 (7): 1371-85.

14. Hara AK, Leighton JA, Heigh RI, et al. "Crohn disease of the small bowel: Preliminary comparison among CT enterography, capsule Colonoscopy, small-Bowel Follow-through, and ileoscopy". Radiology 2005; 238 (1): 128-34.

15. Jenkins D, Balsitis M, Gallivan S, et al. Guidelines for the initial biopsy diagnosis of suspected chronic idiopathic inflammatory bowel disease. The British Society of Gastroenterology Initiative. J Clin Pathol 1997; 50:93-105.

16. Goldman H. Colonic biopsy in inflammatory bowel disease. Surg Pathol 1991; 4:3-24.

17. Santos J, Guilarte M, Alonso C, et al . Pathogenesis of irritable bowel syndrome: The mast cell connection.Scand J Gastroenterol 2005; 40:129-40.

18. Bouma G, StroberW. The immunological and genetic basis of inflammatory bowel disease. Nat Rev Immunol 2003; 3:521-33.

19. Wassell J, Dolwani S, Metzner M,et al.Faecal calprotectin: a new marker for crohn's disease? Ann Clin Biochem 2004; 41:230 -2.

20. Konikoff M, Denson LA. Role of fecal calprotectin as a biomarker of intestinal inflammation in inflammatory bowel disease. I B D 2006; 12:52434.

21. Tibble JA, Sigthorsson G, Foster R, et al. Use of surrogate markers of inflammation and Rome criteria to distinguish organic from nonorganic intestinal disease. Gastroenterology. 2002; 123:450-60.

22. Jun-Ying Xiang, Qin Ouyang, Guo-Dong Li, et al. Clinical value of fecal calprotectin in determining disease activity of ulcerative colitis. $W J$ Gastroenterol 2008 January 7; 14(1): 53-57 